\title{
Dust-ion-acoustic rogue waves in presence of non-extensive non-thermal electrons
}

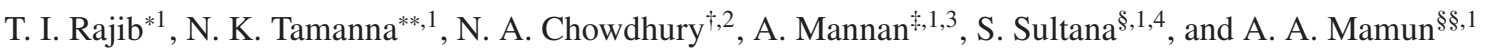 \\ ${ }^{1}$ Department of Physics, Jahangirnagar University, Savar, Dhaka-1342, Bangladesh \\ ${ }^{2}$ Plasma Physics Division, Atomic Energy Centre, Dhaka-1000, Bangladesh \\ ${ }^{3}$ Institut für Mathematik, Martin Luther Universität Halle-Wittenberg, D-06099 Halle, Germany \\ ${ }^{4}$ Lehrstuhl Theoretische Physik IV, Ruhr Universität Bochum, 44780 Bochum, Germany \\ e-mail: ${ }^{*}$ tirajibphys@gmail.com, ${ }^{* *}$ tamanna1995phy@gmail.com, ${ }^{\dagger}$ nurealam1743phy@gmail.com, \\ ¥abdulmannan@juniv.edu, ${ }^{\S}$ ssultana@juniv.edu, ${ }^{\S}$ mamun_phys@juniv.edu,
}

\begin{abstract}
Dust-ion-acoustic (DIA) rogue waves (DIARWs) are investigated in a three components dusty plasma system containing inertialess electrons featuring non-thermal non-extensive distribution as well as inertial warm ions and negative dust grains. A nonlinear Schrödinger equation (NLSE), which governs the conditions of the modulational instability (MI) of DIA waves (DIAWs), is obtained by using the reductive perturbation method. It has been observed from the numerical analysis of NLSE that the plasma system supports both modulationally stable domain in which dispersive and nonlinear coefficients of the NLSE have same sign and unstable domain in which dispersive and nonlinear coefficients of the NLSE have opposite sign, and also supports the DIARWs only in the unstable domain. It is also observed that the basic features (viz. stability of the DIAWs, MI, growth rate, amplitude, and 'width of the DIARWs, etc.) are significantly modified by the related plasma parameters (viz. dust charge state, number density of electron and ion, non-extensive parameter q, and non-thermal parameter $\alpha$, etc.). The present study is useful for understanding the mechanism of the formation of DIARWs in the laboratory and space environments where inertialess mixed distributed electrons can exist with inertial ions and dust grains.
\end{abstract}

'Keywords: NLSE, Modulational instability, Dust-ion-acoustic waves, Rogue waves.

\section{Introduction}

The investigation of plasma system containing massive dust grains has a great importance due to its wide applications in laboratory and space dusty plasma medium (DPM) [1, 2, 3, 4, 4, 6, 7]. Dust grains can be either negatively/positively charged massive object, if they are injected into any plasma system [3, 4], depending on the ambient environments. Such kind of highly charged and extremely massive dust grains can modify the normal plasma modes. Among the modified modes, the dust-ion-acoustic (DIA) waves (DIAWs), which theoretically proposed by Shukla and Silin [1] and experimentally confirmed by Barkan et al. [2], has received much attention. Significant research efforts have been devoted in investigating the characteristics of DIAWs in the pair-ion DPM (PIDPM) [5], and degenerate DPM [5, 6]. Misra [5] studied the nonlinear propagation of DIA shocks waves in a quantum PIDPM, and numerically discussed the stationary shock solutions. Duha et al. [6] considered a Multi-ion DPM, and observed both the solitary and shocks structures. Sahu and Tribeche [7] reported the electrostatic dust-acoustic (DA) solitary and shock structures in DPM having non-thermal plasma species.

The nonlinear features of plasma waves are rigorously affected by the velocity distribution function of the plasma particles, and the Maxwellian velocity distribution is one of the most commonly used velocity distribution functions in plasma physics. The space observations [8] and laboratory experiments
[9] indicated the presence of particles which deviates from the Maxwellian distribution. These findings lead to the modification of the Boltzmann-Gibbs-Shannon entropy which has been recognized by Renyi [10] and successively proposed by Tsallis [11]. The index $q$ in the non-extensive $q$-distribution underpins the generalized Tsallis entropy, and is connected to the dynamics of the long range interacting systems, and also measures the degree of its non-extensivity of the plasma species, and has been successfully applied to explain the intrigue mechanism of a number of complex plasma situations [12, 13]. On the other hand, Cairns et al. [14 introduced a distribution to describe the dynamics of the highly energetic tails, in which a parameter $\alpha$ can measure the deviation of the plasma species from the Maxwellian velocity distribution function, in space plasma environments [15, 16]. Paul and Bandyopadhyay [15] investigated DIA solitary waves in a multi-component DPM having non-thermal electrons. Tasnim et al. [16] examined the effects of non-thermal plasma species on the the formation of DA Gardner solitons in DPM. A number of authors have considered a hybrid Cairn-Tsallis/non-thermal non-extensive distribution $\lfloor 17,18,19,20,21,22,23,24$ for analyzing the nonlinear properties of the plasma medium and found that this hybrid Cairn-Tsallis/non-thermal non-extensive distribution is suitable to provide a better picture which coincides with a wide range of the space observational data [25, 26].

Rogue wave (RW) [27, 28, 29], which governs by the ra- 
tional solution of the standard nonlinear Schrödinger equation (NLSE) [27, 28, 29, 30, 31, 32, 33, 34, 35, 36] in a dispersive medium, was firstly observed in the ocean. It is a rare, shortlived, and high-energy event with amplitude much higher than the average wave crests around it [27]. The usual feature of a RW is that it will appear suddenly and increase up to a very high amplitude with the exponential growth, and finally disappear without a trace [28]. Recently, a number of authors have employed the reductive perturbation method (RPM) to establish the NLSE for the investigation of modulational instability (MI) of the DA waves (DAWs) and also the formation of DA RWs (DARWs) in various plasma system [33, 34, 35, 36]. Bains et al. [29] theoretically and numerically analyzed the effect of non-extensivity of electrons and ions on the MI criteria of DAWs. Rahman et al. [33] investigated the MI conditions of the DAWs in presence of the non-thermal plasma species, and observed that the maximum value of the MI growth rate increases with the non-extensive parameter $q$, and also found that the amplitude and width of the RWs increase with an increase in the value of the non-thermal parameter $\alpha$. El-Labany et al. [34] have considered a three components DPM having non-thermal plasma species for investigation of the DARWs, and found that the nonlinearity of the DPM increases with $\alpha$. Hassan et al. [35] analyzed theoretically and numerically the instability conditions of the ion-acoustic waves in a non-thermal plasma, and highlighted that the non-thermality of the electrons enhances the nonlinearity of the plasma medium as well as the height and thickness of the RWs. Misra and Chowdhury [36] considered a three components plasma model having non-thermal Cairn's distributed ions, and observed that the envelope solitons are significantly modified by the presence of non-thermal ions.

In DAWs, the moment of inertia is provided by the dust grains and restoring force is provided by the thermal pressure of the ions and electrons. On the other hand in DIAWs, the moment of inertia is provided by the ions and restoring force is provided by the thermal pressure of the electrons in presence of immobile dust grains. The mass and charge of the dust grains are considerably larger than the ions while the mass and charge of the ion are considerably larger than the electron. It may be noted here that in the DIAWs, if anyone consider the pressure term of the ions then it is important to be considered the moment of inertia of the ions along with the dust grains in presence of inertialess electrons. This means that the consideration of the pressure term of the ions highly contributes to the moment of inertia along with inertial dust grains to generate DIAWs in a DPM having inertialess electrons. In the present work, we are interested to investigate the nonlinear propagation of DIARWs in which the moment of inertia is provided by the inertial warm ions and negatively charged dust grains and the restoring force is provided by the thermal pressure of the inertialess electrons in a three components DPM (dusts-ions-electrons) by using the standard NLSE.

The layout of the manuscript is as follows: The governing equations describing our plasma model are presented in Section 2. The derivation of a NLSE is given in Section 3. The MI and RWs are discussed in Section 4 Numerical analysis is presented in Section 5. A conclusion is provided in Section6.

\section{Governing Equations}

We consider a three components DPM comprising of inertial positively charged warm ion (charge $q_{i}=Z_{i} e$ and mass $m_{i}$ ) and inertial negatively charged dust grains (charge $q_{d}=-Z_{d} e$ and mass $m_{d}$ ) as well as inertialess non-thermal non-extensive electron (charge $q_{e}=-e$; mass $m_{e}$ ); where $Z_{i}\left(Z_{d}\right)$ is the number of protons (electrons) residing onto the ion (dust grains) surface, and $e$ is the magnitude of the charge of an electron. Overall, the charge neutrality condition for our plasma model can be written as $Z_{i} n_{i 0}=Z_{d} n_{d 0}+n_{e 0}$. Now, the normalized governing equations of the DIAWs can be written as

$$
\begin{aligned}
& \frac{\partial n_{d}}{\partial t}+\frac{\partial}{\partial x}\left(n_{d} u_{d}\right)=0, \\
& \frac{\partial u_{d}}{\partial t}+u_{d} \frac{\partial u_{d}}{\partial x}=\rho_{1} \frac{\partial \phi}{\partial x}, \\
& \frac{\partial n_{i}}{\partial t}+\frac{\partial}{\partial x}\left(n_{i} u_{i}\right)=0, \\
& \frac{\partial u_{i}}{\partial t}+u_{i} \frac{\partial u_{i}}{\partial x}+\rho_{2} n_{i} \frac{\partial n_{i}}{\partial x}=-\frac{\partial \phi}{\partial x}, \\
& \frac{\partial^{2} \phi}{\partial x^{2}}+n_{i}=\rho_{3} n_{e}+\left(1-\rho_{3}\right) n_{d},
\end{aligned}
$$

where $n_{d}\left(n_{i}\right)$ is the dust (ion) number density normalized by its equilibrium value $n_{d 0}\left(n_{i 0}\right) ; u_{d}\left(u_{i}\right)$ is the dust (ion) fluid speed normalized by the ion-acoustic wave speed $C_{i}=\left(Z_{i} k_{B} T_{e} / m_{i}\right)^{1 / 2}$ with $T_{e}$ being the non-thermal non-extensive electron temperature, and $k_{B}$ being the Boltzmann constant; $\phi$ is the electrostatic wave potential normalized by $k_{B} T_{e} / e$; the time and space variables are normalized by $\omega_{p i}^{-1}=\left(m_{i} / 4 \pi Z_{i}^{2} e^{2} n_{i 0}\right)^{1 / 2}$ and $\lambda_{D i}=\left(k_{B} T_{e} / 4 \pi Z_{i} e^{2} n_{i 0}\right)^{1 / 2}$, respectively. The pressure term of the ion is recognized as $P_{i}=P_{i 0}\left(N_{i} / n_{i 0}\right)^{\gamma}$ with $P_{i 0}=n_{i 0} k_{B} T_{i}$ being the equilibrium pressure of the ion, and $T_{i}$ being the temperature of warm ion, and $\gamma=(N+2) / N$ (where $N$ is the degree of freedom and for one-dimensional case $N=1$, then $\gamma=3)$. Other plasma parameters are $\rho_{1}=\rho \mu, \rho=Z_{d} / Z_{i}$, $\mu=m_{i} / m_{d}, \rho_{2}=3 T_{i} / Z_{i} T_{e}$, and $\rho_{3}=n_{e 0} / Z_{i} n_{i 0}$, etc. Now, the expression for the number density of non-thermal non-extensive electrons following the non-thermal non-extensive distribution [17, 18, 19, 20, 21] can be written as

$$
n_{e}=\left[1+A \phi+B \phi^{2}\right] \times[1+(q-1) \phi]^{\frac{(q+1)}{2(q-1)}},
$$

where the parameter $q$ stands for the strength of non-extensive system and the coefficients $A$ and $B$ are defined by $A=$ $-16 q \alpha /\left(3-14 q+15 q^{2}+12 \alpha\right)$ and $B=-A(2 q-1)$. Here $\alpha$ is a parameter determining the number of non-thermal electrons in the model. Williams et al. [21] discussed the range and the validity of $(q, \alpha)$ for solitons. In the limiting case $(q \rightarrow 1$ and $\alpha=0$ ), the above distribution reduces to the well-known Maxwell-Boltzmann velocity distribution. For $(q \rightarrow 1$ and $\alpha \neq 0$ ), the above distribution reduces to Cairn distribution.

Now, by substituting Eq. (6) into Eq. (5), and expanding up to third order of $\phi$, we get

$$
\begin{aligned}
\frac{\partial^{2} \phi}{\partial x^{2}}+n_{i}= & \rho_{3}+\left(1-\rho_{3}\right) n_{d}+S_{1} \phi \\
& +S_{2} \phi^{2}+S_{3} \phi^{3}+\cdots,
\end{aligned}
$$


where

$$
\begin{aligned}
& S_{1}=\left[2 \rho_{3} A+\rho_{3}(q+1)\right] / 2, \\
& S_{2}=\left[8 \rho_{3} B+4 \rho_{3} A(q+1)-\rho_{3}(q+1)(q-3)\right] / 8, \\
& S_{3}=\left[24 \rho_{3} B(q+1)-6 \rho_{3} A(q+1)(q-3)+M_{1}\right] / 48,
\end{aligned}
$$

with $M_{1}=\rho_{3}(q+1)(q-3)(3 q-5)$. The terms containing $S_{1}, S_{2}$, and $S_{3}$ in Eq. (7) are due to the contribution of the non-thermal non-extensive electrons.

\section{Derivation of the NLSE}

To study the MI of the DIAWs, we want to derive the NLSE by employing the RPM and for that case, first we can write the stretched co-ordinates in the form

$$
\begin{aligned}
& \xi=\epsilon\left(x-v_{g} t\right), \\
& \tau=\epsilon^{2} t,
\end{aligned}
$$

where $v_{g}$ is the group speed and $\epsilon(0<\epsilon<1)$ is a small parameter measuring the weakness of the dispersion. Then we can write the dependent variables as

$$
\begin{aligned}
& n_{d}=1+\sum_{m=1}^{\infty} \epsilon^{m} \sum_{l=-\infty}^{\infty} n_{d l}^{(m)}(\xi, \tau) \mathrm{e}^{i l(k x-\omega t)}, \\
& u_{d}=\sum_{m=1}^{\infty} \epsilon^{m} \sum_{l=-\infty}^{\infty} u_{d l}^{(m)}(\xi, \tau) \mathrm{e}^{i l(k x-\omega t)}, \\
& n_{i}=1+\sum_{m=1}^{\infty} \epsilon^{m} \sum_{l=-\infty}^{\infty} n_{i l}^{(m)}(\xi, \tau) \mathrm{e}^{i l(k x-\omega t)}, \\
& u_{i}=\sum_{m=1}^{\infty} \epsilon^{m} \sum_{l=-\infty}^{\infty} u_{i l}^{(m)}(\xi, \tau) \mathrm{e}^{i l(k x-\omega t)}, \\
& \phi=\sum_{m=1}^{\infty} \epsilon^{m} \sum_{l=-\infty}^{\infty} \phi_{l}^{(m)}(\xi, \tau) \mathrm{e}^{i l(k x-\omega t)},
\end{aligned}
$$

where $k$ and $\omega$ are real variables representing the carrier wave number and frequency, respectively. The derivative operators can be written as

$$
\begin{aligned}
& \frac{\partial}{\partial x} \rightarrow \frac{\partial}{\partial x}+\epsilon \frac{\partial}{\partial \xi}, \\
& \frac{\partial}{\partial t} \rightarrow \frac{\partial}{\partial t}-\epsilon v_{g} \frac{\partial}{\partial \xi}+\epsilon^{2} \frac{\partial}{\partial \tau} .
\end{aligned}
$$

Now, by substituting Eqs. (8)-(16) into Eqs. (11)-(4), and (7), and collecting the terms containing $\epsilon$, the first order (when $m=$ 1 with $l=1$ ) reduced equations can be written as

$$
\begin{aligned}
& n_{d 1}^{(1)}=-\frac{\rho_{1} k^{2}}{\omega^{2}} \phi_{1}^{(1)}, \\
& u_{d 1}^{(1)}=-\frac{\rho_{1} k}{\omega} \phi_{1}^{(1)}, \\
& n_{i 1}^{(1)}=\frac{k^{2}}{\omega^{2}-\rho_{2} k^{2}} \phi_{1}^{(1)}, \\
& u_{i 1}^{(1)}=\frac{\omega k}{\omega^{2}-\rho_{2} k^{2}} \phi_{1}^{(1)}, \\
& n_{i 1}^{(1)}=\left(1-\rho_{3}\right) n_{d 1}^{(1)}+k^{2} \phi_{1}^{(1)}+S_{1} \phi_{1}^{(1)},
\end{aligned}
$$
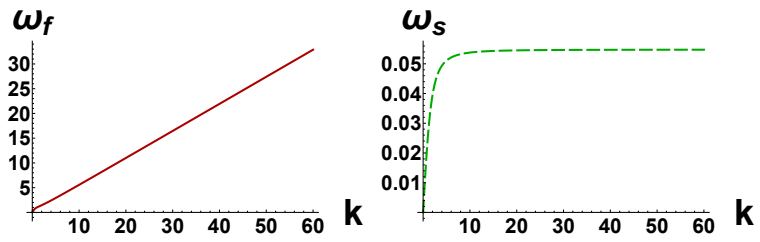

Figure 1: $\omega_{f}$ vs $k$ (left panel) and $\omega_{s}$ vs $k$ (right panel) when other plasma parameters are $\alpha=0.5, q=1.6, \mu=2 \times 10^{-6}, \rho=3 \times 10^{3}, \rho_{2}=0.3$, and $\rho_{3}=0.5$.

these relation provides the dispersion relation for DIAWs

$\omega^{2}=\frac{M_{2} k^{2} \pm k^{2} \sqrt{M_{2}^{2}-4 M_{3} M_{4}}}{2 M_{3}}$,

where $M_{2}=1+\rho_{1}-\rho_{1} \rho_{3}+\rho_{2} k^{2}+\rho_{2} S_{1}, M_{3}=k^{2}+S_{1}$, and $M_{4}=\rho_{1} \rho_{2}-\rho_{1} \rho_{2} \rho_{3}$. In Eq. (22), to get real and positive values of $\omega$, the condition $M_{2}^{2}>4 M_{3} M_{4}$ should be satisfied. The positive and negative signs in Eq. (22) corresponds to the fast $\left(\omega_{f}\right)$ and slow $\left(\omega_{s}\right)$ DIA modes. The fast DIA mode corresponds to the case in which both inertial dust and ion components oscillate in phase with the inertialess electrons. On the other hand, the slow DIA mode corresponds to the case in which only one of the inertial components oscillates in phase with inertialess electrons, but the other inertial component oscillates in antiphase with them [37, 38]. We have numerically analyzed the fast and slow DIA modes in Fig. 11 in presence of non-thermal non-extensive electrons ( $\alpha=0.5$ and $q=1.6$ ). Figure 1 (left panel) indicates that the frequency of the DIAWs can be higher than ion-plasma frequency. In this regard, we note that in absence of dust, the frequency of the ion-acoustic waves is always less than the ion-plasma or ion-Langmuir frequency. However, the phase speed of the DIAWs increases with the magnitude of the dust charge $\left(Z_{d}\right)$ and dust number density $\left(n_{d 0}\right)$. This is due to the extra space charge electric field created by the highly negative charged dust grains. This is theoretically predicted by Shukla and Silin [1] and experimentally observed by Barkan et al. [2]. Thus, as the magnitude of the dust charge $\left(Z_{d}\right)$ or dust number density $\left(n_{d 0}\right)$ increases, the frequency of the DIAWs increases, even it can exceed the ion-plasma or ion-Langmuir frequency. On the other hand, the dispersion curve of slow DIA mode shown in Fig. 1(right panel) clearly indicates that the frequency of the slow DIA mode is always less than the ion-plasma or ion-Langmuir frequency even in presence of highly negatively charged dust. The second order equations (when $m=2$ with $l=1$ ) are given by

$$
\begin{aligned}
& n_{d 1}^{(2)}=-\frac{\rho_{1} k^{2}}{\omega^{2}} \phi_{1}^{(2)}-\frac{2 i k \rho_{1}\left(v_{g} k-\omega\right)}{\omega^{3}} \frac{\partial \phi_{1}^{(1)}}{\partial \xi}, \\
& u_{d 1}^{(2)}=-\frac{\rho_{1} k}{\omega} \phi_{1}^{(2)}-\frac{i \rho_{1}\left(v_{g} k-\omega\right)}{\omega^{2}} \frac{\partial \phi_{1}^{(1)}}{\partial \xi}, \\
& n_{i 1}^{(2)}=\frac{k^{2}}{\omega^{2}-\rho_{2} k^{2}} \phi_{1}^{(2)}+\frac{2 i k \omega\left(v_{g} k-\omega\right)}{\left(\omega^{2}-\rho_{2} k^{2}\right)^{2}} \frac{\partial \phi_{1}^{(1)}}{\partial \xi}, \\
& u_{i 1}^{(2)}=\frac{k \omega}{\omega^{2}-\rho_{2} k^{2}} \phi_{1}^{(2)}+\frac{i\left(v_{g} k-\omega\right)\left(\omega^{2}+\rho_{2} k^{2}\right)}{\left(\omega^{2}-\rho_{2} k^{2}\right)^{2}} \frac{\partial \phi_{1}^{(1)}}{\partial \xi},
\end{aligned}
$$


with the compatibility condition

$v_{g}=\frac{\rho_{1} \rho_{3} \omega^{5}-\rho_{1} \omega^{5}+2 \rho_{1} \rho_{2} \omega^{3} k^{2}+M_{5}}{\rho_{1} \rho_{3} \omega^{4} k-\rho_{1} \omega^{4} k+\rho_{1} \rho_{2}^{2} \rho_{3} k^{5}+M_{6}}$,

where

$$
\begin{aligned}
M_{5}= & -2 \rho_{1} \rho_{2} \rho_{3} \omega^{3} k^{2}-\omega \rho_{1} \rho_{2}^{2} k^{4}+\omega \rho_{1} \rho_{3} \rho_{2}^{2} k^{4}-\omega^{5} \\
& +\omega^{7}-2 \rho_{2} \omega^{5} k^{2}+\rho_{2}^{2} \omega^{3} k^{4}, \\
M_{6}= & 2 \rho_{1} \rho_{2} \omega^{2} k^{3}-2 \rho_{1} \rho_{2} \rho_{3} \omega^{2} k^{3}-\rho_{1} \rho_{2}^{2} k^{5}-\omega^{4} k .
\end{aligned}
$$

The coefficients of the $\epsilon$ when $m=2$ with $l=2$ provides the second order harmonic amplitudes which are found to be proportional to $\left|\phi_{1}^{(1)}\right|^{2}$

$n_{d 2}^{(2)}=S_{4}\left|\phi_{1}^{(1)}\right|^{2}$,

$u_{d 2}^{(2)}=S_{5}\left|\phi_{1}^{(1)}\right|^{2}$,

$n_{i 2}^{(2)}=S_{6}\left|\phi_{1}^{(1)}\right|^{2}$,

$u_{i 2}^{(2)}=S_{7}\left|\phi_{1}^{(1)}\right|^{2}$,

$\phi_{2}^{(2)}=S_{8}\left|\phi_{1}^{(1)}\right|^{2}$,

where

$$
\begin{aligned}
& S_{4}=\frac{3 \rho_{1}^{2} k^{4}-2 \rho_{1} \omega^{2} k^{2} S_{8}}{2 \omega^{4}}, \\
& S_{5}=\frac{\rho_{1}^{2} k^{3}-2 \rho_{1} \omega^{2} k S_{8}}{2 \omega^{3}}, \\
& S_{6}=\frac{2 k^{2} S_{8}\left(\omega^{2}-\rho_{2} k^{2}\right)^{2}+k^{4}\left(\rho_{2} k^{2}+3 \omega^{2}\right)}{2\left(\omega^{2}-\rho_{2} k^{2}\right)^{3}}, \\
& S_{7}=\frac{\omega S_{6}\left(\omega^{2}-\rho_{2} k^{2}\right)-\omega k^{4}}{k\left(\omega^{2}-\rho_{2} k^{2}\right)^{2}}, \\
& S_{8}=\frac{k^{4} \omega^{4}\left(\rho_{2} k^{2}+3 \omega^{2}\right)-\left(3 \rho_{1}^{2} k^{4}+2 \omega^{4} S_{2}\right)\left(\omega^{2}-\rho_{2} k^{2}\right)^{3}}{6 k^{2} \omega^{4}\left(\omega^{2}-\rho_{2} k^{2}\right)^{3}} .
\end{aligned}
$$

When $m=3$ with $l=0$ and $m=2$ with $l=0$ lead to zeroth harmonic modes as follows

$n_{d 0}^{(2)}=S_{9}\left|\phi_{1}^{(1)}\right|^{2}$,

$u_{d 0}^{(2)}=S_{10}\left|\phi_{1}^{(1)}\right|^{2}$,

$n_{i 0}^{(2)}=\left.S_{11} \phi_{1}^{(1)}\right|^{2}$,

$u_{i 0}^{(2)}=S_{12}\left|\phi_{1}^{(1)}\right|^{2}$,

$\phi_{0}^{(2)}=S_{13}\left|\phi_{1}^{(1)}\right|^{2}$,

where

$$
\begin{aligned}
& S_{9}=\frac{\rho_{1}^{2} k^{2} \omega+2 v_{g} \rho_{1}^{2} k^{3}-\rho_{1} S_{13} \omega^{3}}{v_{g}^{2} \omega^{3}}, \\
& S_{10}=\frac{k^{2} \rho_{1}^{2}-\rho_{1} S_{13} \omega^{2}}{v_{g} \omega^{2}}, \\
& S_{11}=-\frac{S_{13}\left(\omega^{2}-\rho_{2} k^{2}\right)^{2}+k^{2}\left(\rho_{2} k^{2}+\omega^{2}+2 \omega v_{g} k\right)}{\left(\rho_{2}-v_{g}^{2}\right)\left(\omega^{2}-\rho_{2} k^{2}\right)^{2}}, \\
& S_{12}=\frac{v_{g} S_{11}\left(\omega^{2}-\rho_{2} k^{2}\right)^{2}-2 \omega k^{3}}{\left(\omega^{2}-\rho_{2} k^{2}\right)^{2}}, \\
& S_{13}=\frac{2 S_{2} v_{g}^{2} \omega^{3}\left(\rho_{2}-v_{g}^{2}\right)\left(\omega^{2}-\rho_{2} k^{2}\right)^{2}+M_{7}}{\omega^{3}\left(\omega^{2}-\rho_{2} k^{2}\right)^{2} \times M_{8}},
\end{aligned}
$$

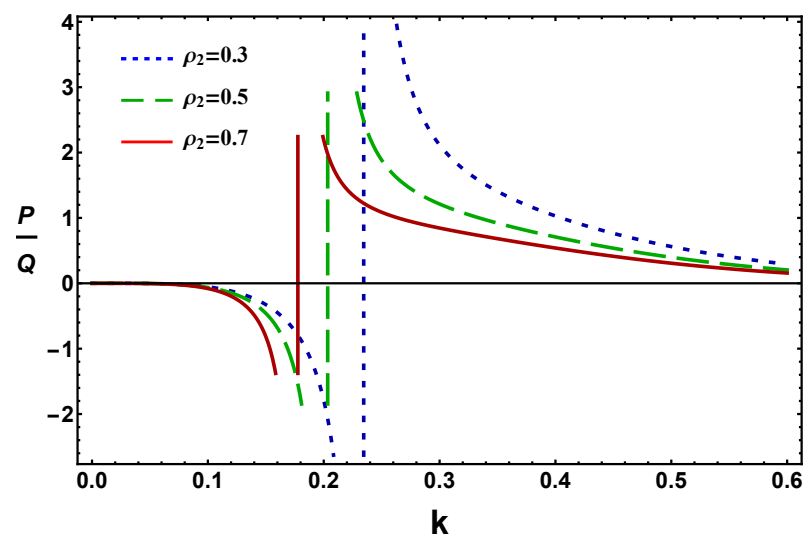

Figure 2: The variation of $P / Q$ with $k$ for different values of $\rho_{2}$ when other plasma parameters are $\alpha=0.5, q=1.6, \mu=2 \times 10^{-6}, \rho=3 \times 10^{3}, \rho_{3}=0.5$, and $\omega_{f}$.

with

$$
\begin{aligned}
M_{7}= & k^{2} v_{g}^{2} \omega^{3}\left(\rho_{2} k^{2}+\omega^{2}\right)+2 k^{3} v_{g}^{3} \omega^{4} \\
& +\rho_{1}^{2} k^{2} \omega\left(1-\rho_{3}\right)\left(\rho_{2}-v_{g}^{2}\right)\left(\omega^{2}-\rho_{2} k^{2}\right) \\
& +2 v_{g} \rho_{1}^{2} k^{3}\left(1-\rho_{3}\right)\left(\rho_{2}-v_{g}^{2}\right)\left(\omega^{2}-\rho_{2} k^{2}\right) \\
M_{8}= & \rho_{1}\left(1-\rho_{3}\right)\left(\rho_{2}-v_{g}^{2}\right)-v_{g}^{2}-v_{g}^{2} S_{1}\left(\rho_{2}-v_{g}^{2}\right) .
\end{aligned}
$$

Finally, the third harmonic modes when $m=3$ and $l=1$ with the help of (17)-37), give a set of equations, which can be reduced to the standard NLSE:

$$
i \frac{\partial \Phi}{\partial \tau}+P \frac{\partial^{2} \Phi}{\partial \xi^{2}}+Q|\Phi|^{2} \Phi=0
$$

where $\Phi=\phi_{1}^{(1)}$ for simplicity. In equation (38), dispersion coefficient $P$ can be written as

$$
P=-\frac{2 k v_{g} \omega^{5}\left(\omega v_{g}-\rho_{2} k\right)\left(\omega-v_{g} k\right)+M_{9}}{\omega\left(\omega^{2}-\rho_{2} k^{2}\right) \times M_{10}},
$$

where

$$
\begin{aligned}
M_{9}= & \rho_{1}\left(1-\rho_{3}\right)\left(\omega-v_{g} k\right)\left(4 v_{g} k-\omega\right)\left(\omega^{2}-\rho_{2} k^{2}\right)^{3} \\
& +\omega^{4}\left(\omega^{2}-\rho_{2} k^{2}\right)^{3}-\omega^{4}\left(\omega-v_{g} k\right)^{2}\left(\omega^{2}+\rho_{2} k^{2}\right), \\
M_{10}= & 2 \omega^{4} k^{2}+2 \rho_{1} k^{2}\left(1-\rho_{3}\right)\left(\omega^{2}-\rho_{2} k^{2}\right)^{2},
\end{aligned}
$$

and also the nonlinear coefficient $Q$ can be written as

$$
Q=\frac{M_{11}}{2 \omega^{4} k^{2}+2 \rho_{1} k^{2}\left(1-\rho_{3}\right)\left(\omega^{2}-\rho_{2} k^{2}\right)^{2}},
$$

where

$$
\begin{aligned}
M_{11}= & 3 \omega^{3}\left(\omega^{2}-\rho_{2} k^{2}\right)^{2} S_{3} \\
& +2 \omega^{3}\left(\omega^{2}-\rho_{2} k^{2}\right)^{2} S_{2}\left(S_{8}+S_{13}\right) \\
& -\omega \rho_{1} k^{2}\left(1-\rho_{3}\right)\left(S_{4}+S_{9}\right)\left(\omega^{2}-\rho_{2} k^{2}\right)^{2} \\
& -2 \rho_{1} k^{3}\left(1-\rho_{3}\right)\left(S_{5}+S_{10}\right)\left(\omega^{2}-\rho_{2} k^{2}\right)^{2} \\
& -2 \omega^{4} k^{3}\left(S_{7}+S_{12}\right)-\omega^{3}\left(\omega^{2} k^{2}+\rho_{2} k^{4}\right)\left(S_{6}+S_{11}\right) .
\end{aligned}
$$

It is interesting that the dispersive coefficient $P$ and nonlinear coefficient $Q$ of the NLSE (38) are function of various plasma parameters such as carrier wave number $k$, the ratio of positive 


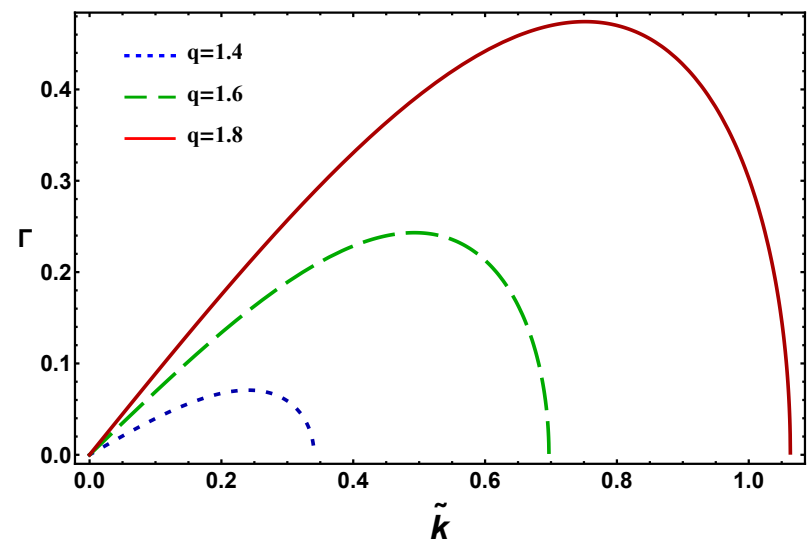

Figure 3: The variation of $\Gamma$ with $\widetilde{k}$ for different values of $q$ when other plasma parameters are $\alpha=0.5, \mu=2 \times 10^{-6}, \rho=3 \times 10^{3}, \rho_{2}=0.3, \rho_{3}=0.5, k=0.4$, $\Phi_{0}=0.5$, and $\omega_{f}$.

ion mass to negative dust mass (via $\mu$ ), the ratio of negative dust charge state to positive ion charge state (via $\rho$ ), ion temperature to electron temperature times the charge state of the positive ion (via $\rho_{2}$ ), the number density of electron to the number density of positive ion times the charge state of the positive ion (via $\rho_{3}$ ), the non-thermality (via $\alpha$ ) and non-extensivity (via $q$ ) properties of electrons, etc.

\section{MI and Rogue Waves}

To study the MI of DIAWs, we consider the linear solution of the NLSE (38) in the form $\Phi=\widetilde{\Phi} e^{i Q|\widetilde{\Phi}|^{2} \tau}+$ c.c., where $\widetilde{\Phi}=$ $\widetilde{\Phi}_{0}+\epsilon \widetilde{\Phi}_{1}$ and $\widetilde{\Phi}_{1}=\widetilde{\Phi}_{1,0} e^{i(\widetilde{k} \xi-\widetilde{\omega} \tau)}+c . c$. We note that the amplitude depends on the frequency, and that the perturbed wave number $\widetilde{k}$ and frequency $\widetilde{\omega}$ which are different from $k$ and $\omega$. Now, substituting these into NLSE (38), one can easily obtain the following nonlinear dispersion relation [30, 31, 32, 29, 33.

$$
\widetilde{\omega}^{2}=P^{2} \widetilde{k}^{2}\left(\widetilde{k}^{2}-\frac{2\left|\widetilde{\Phi}_{0}\right|^{2}}{P / Q}\right) .
$$

It is observed here that the ratio $P / Q$ is negative (i.e., $P / Q<0$ ), the DIAWs will be modulationally stable. On the other hand, if the ratio $P / Q$ is positive (i.e., $P / Q>0$ ), the DIAWs will be modulationally unstable. It is obvious from Eq. (39) that the DIAWs becomes modulationally unstable when $\widetilde{k}_{c}>\widetilde{k}$ in the regime $P / Q>0$, where $\widetilde{k}_{c}=\sqrt{2(Q / P)}\left|\widetilde{\Phi}_{0}\right|$. The growth rate $\Gamma$ of the modulationally unstable DIAWs is given by

$$
\Gamma=|P| \widetilde{k}^{2} \sqrt{\frac{\widetilde{k}_{c}^{2}}{\bar{k}^{2}}-1} .
$$

Equation (40) can easily express the nonlinear character of the DPM. The first-order rational solution, in the domain where dispersive and nonlinear coefficients of NLSE have an opposite sign (i.e., $P / Q>0$ ), can be written as [27]

$$
\Phi(\xi, \tau)=\sqrt{\frac{2 P}{Q}}\left[\frac{4(1+4 i P \tau)}{1+16 P^{2} \tau^{2}+4 \xi^{2}}-1\right] e^{2 i P \tau},
$$

The Eq. (41) implies that the concentration of high energy occurs within a small region.

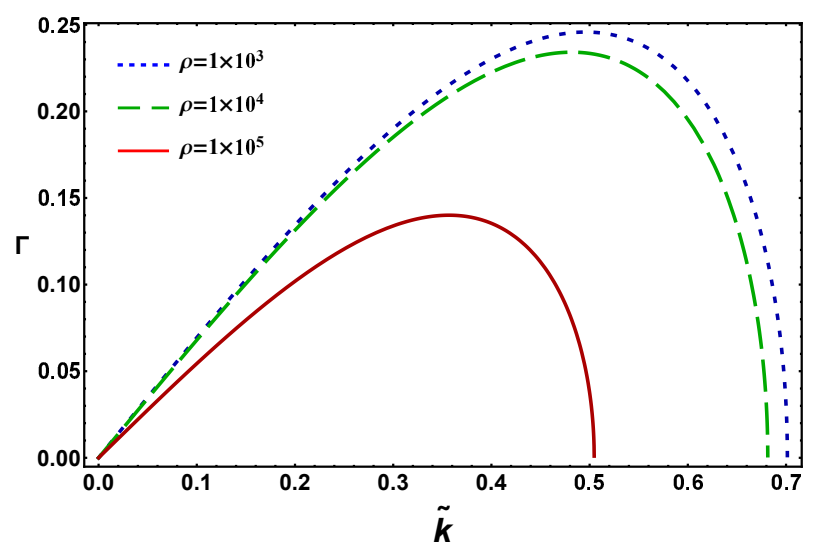

Figure 4: The variation of $\Gamma$ with $\widetilde{k}$ for different values of $\rho$ when other plasma parameters are $\alpha=0.5, q=1.6, \mu=2 \times 10^{-6}, \rho_{2}=0.3, \rho_{3}=0.5, k=0.4$, $\Phi_{0}=0.5$, and $\omega_{f}$.

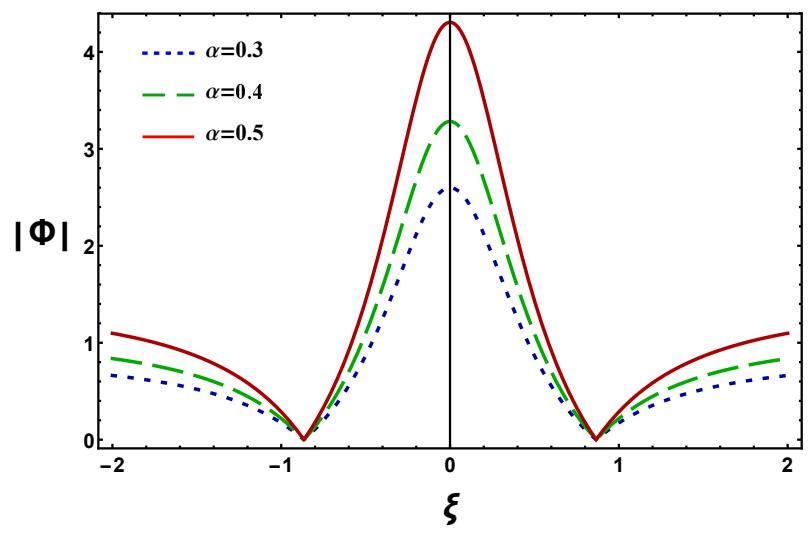

Figure 5: The variation of $|\Phi|$ with $\xi$ for different values of $\alpha$ when other plasma parameters are $q=1.6, \mu=2 \times 10^{-6}, \rho=3 \times 10^{3}, \rho_{2}=0.3, \rho_{3}=0.5, k=0.4$, $\tau=0$, and $\omega_{f}$.

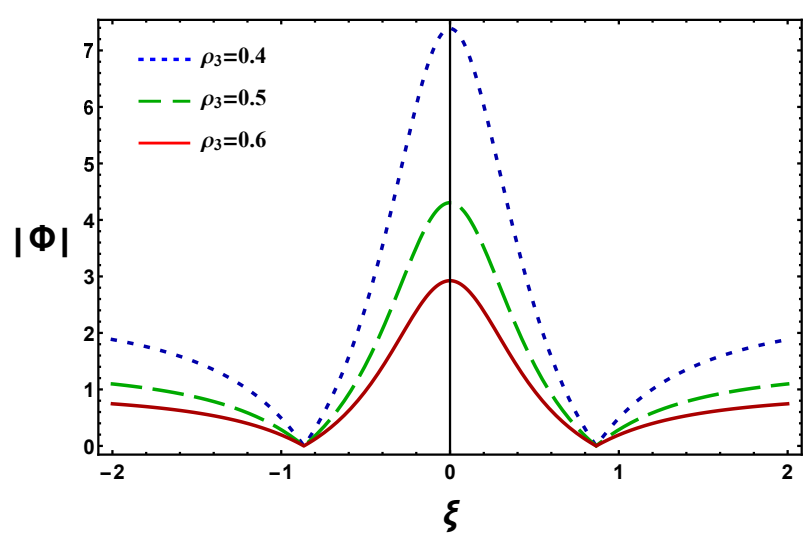

Figure 6: The variation of $|\Phi|$ with $\xi$ for different values of $\rho_{3}$ when other plasma parameters are $\alpha=0.5, q=1.6, \mu=2 \times 10^{-6}, \rho=3 \times 10^{3}, \rho_{2}=0.3$, $k=0.4, \tau=0$, and $\omega_{f}$.

\section{Numerical analysis}

Now, we would like to numerically analyze the stability conditions of the DIAWs in presence of the non-thermal nonextensive electrons. The mass and charge state of the plasma species even their number density are important factors in 


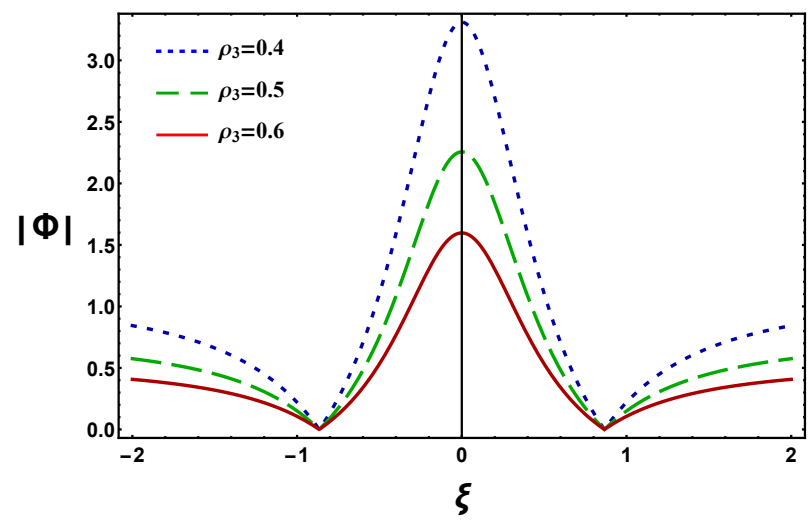

Figure 7: The variation of $|\Phi|$ with $\xi$ for different values of $\rho_{3}$ when other plasma parameters are $\alpha=0, q=1, \mu=2 \times 10^{-6}, \rho=3 \times 10^{3}, \rho_{2}=0.3$, $k=0.4, \tau=0$, and $\omega_{f}$.

recognizing the stability conditions of the DIAWs in DPM $[38,39,40,41,42,43]$. The mass of the dust grains is comparable to the mass of proton. In general picture of the DPM, dust grains are massive (millions to billions times heavier than the protons) and their sizes range from nanometres to millimetres. Dust grains may be metallic, conducting, or made of ice particulate. The size and shape of dust grains will be different, unless they are man-made. The dust grains are millions to billions times heavier than the protons, and typically, a dust grain acquires one thousand to several hundred thousand elementary charges [38, 39, 40, 41, 42, 43]. In this article, we consider three components dusty plasma model having inertial warm positive ions and negative dust grains, and interialess non-thermal nonextensive electrons. It may be noted here that in the DIAWs, if anyone consider the pressure term of the ions then it is important to be considered the moment of inertia of the ions along with the dust grains in presence of inertialess electrons. This means that the consideration of the pressure term of the ions highly contributes to the moment of inertia along with inertial dust grains to generate DIAWs in a DPM having inertialess electrons. In our present analysis, we have considered that $m_{d}=10^{6} m_{i}, Z_{d}=\left(10^{3} \sim 10^{5}\right) Z_{i}$, and $T_{e}=10 T_{i}$.

The variation of $P / Q$ with $k$ for different values of $\rho_{2}$ is shown in Fig. 2 which provides the information about the effects of the temperature of the positive ion $\left(T_{i}\right)$ and negative electron $\left(T_{e}\right)$, and the charge state of the positive ion $Z_{i}$ in presence of non-Maxwellian (i.e., $\alpha=0.5$ and $q=1.6$ ) electrons. The point, in which $P / Q$ curve coincides with the $k$-axis in $P / Q$ versus $k$ graph, is known as threshold/critical wave number $\left(k_{c}\right)$ which divides the unstable domain from stable one for the DIAWs. It is clear from this figure that when $\rho_{2}=0.3,0.5,0.7$, then the corresponding value of $k_{c} \cong 0.24$ (dotted blue curve), 0.20 (dashed green), 0.18 (solid red curve). Both modulationally stable (i.e., for long wavelength) and unstable (i.e., for short wavelength) domains of DIAWs are allowed by non-thermal non-extensive DPM, and if we increase (decrease) in the value of $\rho_{2}$ which can shift the $k_{c}$ to a lower (higher) value of $k$ and this means that the DIAWs will be unstable for small values of $k$ with an increase in the ion temperature while stable for the large values of $k$ with an increase in the electron temperature.

We have numerically analyzed Eq. (40) in Figs. 3 and 4 by using these plasma parameters: $\alpha=0.5, \mu=2 \times 10^{-6}, \rho_{2}=0.3$, $\rho_{3}=0.5, \Phi_{0}=0.5, k=0.4, \omega_{f}$, and have also maintained the assumption $m_{d}=10^{6} m_{i}, Z_{d}=\left(10^{3} \sim 10^{5}\right) Z_{i}$, and $T_{e}=10 T_{i}$ for the depiction of the growth rate of DIAWs. The variation of the $\Gamma$ with $\widetilde{k}$ indicates that initially, the $\Gamma$ increases with $\widetilde{k}$ and becomes maximum for a particular value of $\widetilde{k}$ then again decreases to zero. It can be seen from Fig. 3 that the maximum value of the growth rate increases with the increase in the value of the $q$, and this result is a good agreement with the result of Ref. [33]. The effects of the charge state of the negative dust $\left(Z_{d}\right)$ and positive ion $\left(Z_{i}\right)$ on the growth rate of the MI of DIAWs are displayed in Fig. 4 which indicates that the $\Gamma$ decreases (increases) with $Z_{d}\left(Z_{i}\right)$. Physically, the nonlinearity of the plasma medium as well as maximum value of the $\Gamma$ increases with $Z_{i}$ while the nonlinearity of the plasma medium as well as maximum value of the $\Gamma$ decreases with $Z_{d}$.

The effects of the non-thermality of electrons (via $\alpha$ ) on the amplitude and width of the DIARWs, which can be depicted by using Eq. (41), can be seen from Fig. 5. This figure clearly indicates that the increase in the value of $\alpha$ does not only cause to increase the amplitude of the DIARWs associated DIAWs in the modulationally unstable domain (i.e., $P / Q>0$ ) but also cause to increase the width of the DIARWs associated DIAWs in the modulationally unstable domain (i.e., $P / Q>0$ ). The physics of this result is that increasing $\alpha$ causes to increase the nonlinearity of the plasma medium as well as the amplitude and width of the DIARWs, and this result is a nice agreement with the result of El-Labany et al. [34] and Hassan et al. [35].

The nature of the DIARWs, which can be depicted by using Eq. (41), with the variation of $\rho_{3}$ can be observed from Figs. 6 and 7. It is easy to demonstrate from these two figures that increasing (decreasing) the values of $\rho_{3}$ leads to a decrease (increase) of the amplitude and width of the DIARWs, which indicates that an increase (decrease) of $\rho_{3}$ could shrink (enhance) the amplitude and width of the DIARWs. Actually, the nonlinearity as well as the amplitude and width of the DIARWs associated with DIAWs in the modulationally unstable domain (i.e., $P / Q>0$ ) increase as we increase (decrease) the value of $n_{i 0}\left(n_{e 0}\right)$ for a fixed value of $Z_{i}$. It is interesting from Figs. 6 and 7 that the order of the variation of DIARWs amplitude and width is not depend on the presence of the Maxwellian/nonMaxwellian electrons, and in presence of non-Maxwellian electrons (i.e., $\alpha=0.5$ and $q=1.6$ ), the amplitude of the DIARWs is always greater than the presence of Maxwellian electrons (i.e., $\alpha=0$ and $q=1$ ) for same value of $\rho_{3}$.

Finally, under consideration (i.e., $P / Q>0$ ), the DPM supports the formation of DIARWs associated with DIAWs, and we have already graphically observed the variation of the DIARWs with respect to $\xi$ at $\tau=0$ in Figs. 5 to 7 and these figures clearly describe that the RWs has two minima and one maxima in the potential which is the typical feature of the DIARWs. This property reveals that our plasma system can concentrate a significant amount of DIA wave energy in a relatively small region at $\tau=0$. 


\section{Conclusion}

To conclude, we have considered a DPM having inertial warm positive ions along with inertial negative dust grains, and inertialess electrons featuring non-thermal non-extensive distribution. Two types of DIA modes can be found from the theoretical calculation, and the frequency of the DIAWs increases as the magnitude of the dust charge or dust number density increases even it can exceed the ion-plasma or ion-Languir frequency. We have also numerically observed the characterizes of the MI growth rate of the DIAWs and it is found that the maximum value of the growth rate increases with the increase in the value of the non-extensive parameter and the charge state of the positive ion while decreases with the charge state of negative dust. We have also numerically examined the variation of rational solutions of the NLSE (38) with the plasma parameters $\alpha$ and $\rho_{3}$. It is found that the amplitude and width of the DIARWs increases with $\alpha$ but decreases with $\rho_{3}$. It is interesting fact that in presence of non-Maxwellian electrons, the amplitude of the DIARWs is always greater than the presence of Maxwellian electrons for same value of $\rho_{3}$. It is noted here that the simulation of our present investigation is very important for this new kind DIAWs but beyond the scope of our present work. We, finally, propose that the findings of our present investigation should be useful to understand the nonlinear phenomena (viz. the MI of DIAWs and formation of DIARWs) in a DPM where electrons follow non-extensive non-thermal distribution.

\section{References}

[1] P. K. Shukla and V. Silin, Phys. Scr. 45, 508 (1992).

[2] A. Barkan, N. D. Angelo, and R. L. Merlino, Planet Space Sci. 44, 239 (1996).

[3] M. Rapp, J. Hedin, I. Strelnikova, M. Friedrich, J. Gumbel, and F. Lubken, Geophys. Res. Lett. 32, L23821 (2005).

[4] S. Kim and R. Merlino, Phys. Plasmas 13, 052118 (2006).

[5] A. P. Misra, Phys. Plasmas 16, 033702 (2009).

[6] S. S. Duha, B. Shikha, and A. A. Mamun, Pramana J. Phys. 77, 2 (2011).

[7] B. Sahu and M. Tribeche, Astrophys. Space Sci. 338, 259 (2012).

[8] D. T. Young, J. J. Berthomier, M. Blanc, J. L. Burch, S. Bolton, A. J. Coates, F. J. Cary, R. Goldstein, M. Grande, T. W. Hill, R. E. Johnson, R. A. Baragiola, V. Kelha, D. J. McComas, K. Mursula, E. C. Sittler, K. R. Svenes, K. Szego, P. Tanskanen, M. F. Thomsen, S. Bakshi, B. L. Barraclough, Z. Bebsi, D. Delapp, M. W. Dunlop, J. T. Gosling, L. K. Gilbert, D. Glenn, C. Holmlund, J. M. Illiano, G. R. Lewis, D. R. Linder, S. Maurice, H. J. McAndrews, B. T. Narhein, E. Pallier, D. Reisenfeld, A. M. Rymer, H. T. Smith, R. L. Tokar, J. Vippola, and C. Zinsmeyer, Science 307, 1262 (2005).

[9] R. Lundin, A. Zakharov, R. Pellinen, H. Borg, B. Hultqvist, N. Pissarenko, E. Dubinin, S. Barabash, I. Liede, and H. Koskinen, Nature (London) 341, 609 (1989).

[10] A. Renyi, Acta Math. Hung. 6, 285 (1955).

[11] C. Tsallis, J. Stat. Phys. 52, 479 (1988).

[12] M. Emamuddin, S. Yasmin, and A. A. Mamun, Phys. Plasmas 20, 043705 (2013).

[13] M. Bacha and M. Tribeche, Astrophys. Space Sci. 337, 153 (2012).

[14] R. Cairn, A. A. Mamun, R. Bingham, R. Bostrom, R. Dendy, C. Nairn, and P. K. Shukla, J. Geophys. Res. 22, 2709 (1995).

[15] A. Paul and A. Bandyopadhyay, Astrophys. Space Sci. 361, 361 (2016).

[16] I. Tasnim, M. M. Masud, and A. A. Mamun, Plasma Phys. Rep. 40, 723 (2014).

[17] M. Tribeche, R. Amour, and P. K. Shukla, Phys. Rev. E 85, 037401 (2012).
[18] Y. Y. Wang, J. T. Li, C. Q. Dai, X. F. Chen, and Z. F. Zhang, Phys. Lett. A, 377, 2097 (2013).

[19] S. Guo and L. Mei, Phys. Plasmas 21, 082303 (2014).

[20] S. Guo, L. Mei, and Z. Zhang, Astrophys. Space Sci. 22, 052306 (2015).

[21] G. Williams, I. Kourakis, F. Verheest, and M. A. Hellberg, Phys Rev. E 88, 023103 (2013).

[22] A. El-Depsy and M. M. Selim, Eur. Phys. J. Plus 131, 431 (2016).

[23] R. Amour, M. Tribeche, and P. K. Shukla, Astrophys. Space Sci. 338, 287 (2012).

[24] O. Bouzit, M. Tribeche, and A. S. Bains, Phys. Plasmas, 22, 084506 (2015).

[25] W. F. El-Taibany, N. M. El-Siragy, E. E. Behery, A. A. El-Bendary, R. M. Taha, Chinese J. Phys. 58, 151 (2019).

[26] L. A. Gougam and M. Tribeche, Astrophys. Space Sci. 338, 287 (2012).

[27] N. Akhmediev, A. Ankiewicz, and J. M. Soto-Crespo, Phys. Rev. E 80, 026601 (2009).

[28] D. Kedziora, A. Ankiewicz, and N. Akhmediev, Phys. Rev. E 84, 056611 (2011).

[29] A. S. Bains, M. Tribeche, and C. S. Ng, Astrophys. Space Sci. 343, 621 (2013).

[30] I. Kourakis and P. K. Shukla, Nonlinear Proc. Geophys. 12, 407 (2003).

[31] R. Fedele, Phys. Scr. 65, 502 (2002).

[32] S. Sultana and I. Kourakis, Plasma Phys. Control. Fusion 53, 045003 (2011).

[33] M. H. Rahman, N. A. Chowdhury, A. Mannan, M. Rahman, A. A. Mamun, Chinese J. Phys. 56, 2061 (2018).

[34] S. K. El-Labany, W. F. El-Taibany, and N. A. Zedan, Phys. Plasmas 22, 073702 (2015)

[35] M. Hassan, M. H. Rahman, N. A. Chowdhury, A. Mannan, and A. A. Mamun, Commun. Theor. Phys. 71, 1017 (2019).

[36] A. P. Misra and A. R. Chowdhury, Eur. Phys. J. D 39, 49 (2006).

[37] A. E. Dubinov, Plasma Phys. Rep. 35, 991 (2009).

[38] E. Saberiana, A. Esfandyari-Kalejahib, and M. Afsari-Ghazib, Plasma Phys. Rep. 43, 83 (2017).

[39] R. L. Merlino, J. Plasma Phys. 80, 773 (2014).

[40] P. K. Shukla and B. Eliasson, Phys. Rev. E 86, 046402 (2012).

[41] A. A. Mamun and P. K. Shukla, Phys. Scripta T98, 107 (2002).

[42] M. Shalaby, S. K. El-Labany, E. F. El-Shamy, W. F. El-Taibany, and M. A. Khaled, Phys. Plasmas 16, 123706 (2009).

[43] P. K. Shukla and A. A. Mamun, Introduction to Dusty Plasma Physics (IOP, London, 2002). 